\title{
OPERATOR IDEALS AND OPERATOR SPACES
}

\author{
D. BENJAMIN MATHES AND VERN I. PAULSEN
}

(Communicated by Palle E. T. Jorgensen)

\begin{abstract}
We prove that every full symmetrically normed ideal of operators on a Hilbert space is realizable as the set of completely bounded maps between two homogeneous operator Hilbert spaces, with the c.b. norm equivalent to (but in general not equal to) the symmetric norm. We show that one can have equality of the c.b. norm and the symmetric norm if one leaves the category of operator spaces and passes to a slightly larger category.
\end{abstract}

\section{Preliminaries}

In Gohberg and Krein's text Introduction to the theory of linear nonselfadjoint operators, the reader may find a nice exposition on the theory of symmetrically normed (s.n.) ideals, and since we will be dealing with this theory extensively, we have adopted their text as a reader's companion to this article. Consequently, we have attempted to duplicate their notation wherever possible. We also will frequently use objects that are not defined here but whose definition may be found in [3].

Let $\mathscr{H}$ be a separable Hilbert space, and let $B(\mathscr{H})$ be the Banach algebra of operators on $\mathscr{H}$. In our exposition we will need the following elementary piece of folklore, whose proof we leave to the reader. If $\mathfrak{C}$ is an arbitrary s.n. ideal, we define

$$
\mathfrak{C}^{\prime}=\left\{Y \in B(\mathscr{H}) \mid X Y \in \mathscr{C}_{1} \text { for all } X \in \mathfrak{C}\right\} .
$$

We call $\mathfrak{C}$ a full s.n. ideal in case $\mathfrak{C}^{\prime \prime}=\mathfrak{C}$.

1.1. Lemma. Let $\Phi$ be an s.n. function. If $\Phi$ is equivalent to the maximal s.n. function, then $\mathfrak{C}_{\Phi}^{\prime}=B(\mathscr{H})$. If $\Phi$ is not equivalent to the maximal s.n. function, then $\mathfrak{C}_{\Phi}^{\prime}=\mathfrak{C}_{\Phi^{*}}$.

In addition to the content of [3], we need to assume that the reader is familiar with the basic ideas and definitions of abstract operator spaces and their completely bounded (c.b.) mappings. These basics may be found in [1] or [2]. The essence of our paper is that, as a set, every full symmetrically normed ideal of operators on a Hilbert space is realizable as the set of completely bounded

Received by the editors August 23, 1993 and, in revised form, September 27, 1993.

1991 Mathematics Subject Classification. Primary 47B10; Secondary 46A11, 46A32, 47A30, 46B28, 46B70, 47D50.

The second author was supported in part by an NSF grant. 
maps between two operator Hilbert spaces. However, it may happen that the symmetric norm itself is not realizable as a c.b. norm, even though it is equivalent to one. Consequently, although we are primarily interested in operator spaces, we wish to introduce a larger category of matricially normed spaces where the symmetric norm will be realizable as a c.b. norm. Given a matrically normed space $\left(\mathscr{X},\|\cdot\|_{n}\right)$, we will say that $\left(\mathscr{X},\|\cdot\|_{n}\right)$ is a matrix cross normed space (an m.c.n. space) if it is a matrix normed space that gives rise to a cross norm on $\mathscr{X} \otimes M_{n}$ for each $n$, when $M_{n}$ is regarded with the Hilbert space operator norm. We will refer to $\left(\mathscr{X},\|\cdot\|_{1}\right)$ as the base space. The reader will verify that all operator spaces are m.c.n. spaces, but not conversely. In particular, if $\mathscr{H}_{\text {proj }}$ denotes the m.c.n. space induced by the projective tensor norm, then a calculation reveals that $\left\|e \otimes E_{11}+f \otimes E_{22}\right\|=\sqrt{2}$ whenever $e$ and $f$ are orthogonal unit vectors. Thus $\mathscr{H}_{\text {proj }}$ is not an operator space. We also wish to point out that the class of m.c.n. spaces is considerably smaller than the class of all matrically normed spaces, as defined in [2]. Indeed, all of the $L_{p}$-matricially normed spaces discussed there fail to be m.c.n. spaces as soon as $p<\infty$. In what follows we will be considering many distinct systems of matrix norms over a common base space. Consequently, please remember that we will henceforth use subscripts on norms to distinguish different systems of matrix norms, instead of subscripts indicating dimension as they did in this paragraph.

It sometimes happens that an operator space $\mathscr{X}$ has the property that every bounded map $T: \mathscr{X} \rightarrow \mathscr{X}$ is completely bounded and $|T|=|T|_{\mathrm{cb}}$. For example, $\mathscr{X}_{\min }$ and $\mathscr{X}_{\max }$ have this property (see [1] for the definition of the minimal and maximal operator matrix norms on $\mathscr{X}$ ). Following Pisier's terminology in [6], we will say that an m.c.n. space is homogeneous if it has this property, and we will say that the m.c.n. space $\mathscr{X}$ is Hilbertian if the base space is a Hilbert space.

Let $\mathscr{H}$ be our separable Hilbert space, and assume that $\mathscr{H}_{1}$ and $\mathscr{H}_{2}$ are two homogeneous m.c.n. spaces with base $\mathscr{H}$. We denote the space of completely bounded maps from $\mathscr{H}_{1}$ into $\mathscr{H}_{2}$ by $\mathrm{C} B\left(\mathscr{H}_{1} ; \mathscr{H}_{2}\right)$.

\subsection{Proposition. The space $\mathrm{CB}\left(\mathscr{H}_{1} ; \mathscr{H}_{2}\right)$ is an s.n. ideal in $B(\mathscr{H})$.}

Proof. Let $X \in \mathrm{C} B\left(\mathscr{H}_{1} ; \mathscr{H}_{2}\right)$, and let $A, B \in B(\mathscr{H})$. If we view

$$
A \in \mathrm{CB}\left(\mathscr{H}_{1} ; \mathscr{H}_{1}\right), \quad X \in \mathrm{C} B\left(\mathscr{H}_{1} ; \mathscr{H}_{2}\right), \quad \text { and } \quad B \in \mathrm{C} B\left(\mathscr{H}_{2} ; \mathscr{H}_{2}\right) \text {, }
$$

then the inequality

$$
|A X B|_{\mathrm{cb}} \leq|A|_{\mathrm{cb}}|X|_{\mathrm{cb}}|B|_{\mathrm{cb}}=|A||X|_{\mathrm{cb}}|B|
$$

follows from homogeneity.

Assume that $X$ is a rank one operator, say $X(f)=\langle f, g\rangle h$ for all $f \in \mathscr{H}$. Let $f_{i j} \in \mathscr{H}$ and let $C=\left(\left\langle f_{i j}, g\right\rangle\right)$. Since $\mathscr{H}_{2}$ is an m.c.n. space, we have

$$
\left\|\left(X\left(f_{i j}\right)\right)\right\|=\|h \otimes C\|=\|C\|\|h\| \leq\left\|\left(f_{i j}\right)\right\|\|g\|\|h\| \text {. }
$$

It follows that $\|X\|_{\mathrm{cb}} \leq\|g\|\|h\|=\|X\|$. Since $\left(\mathrm{CB}\left(\mathscr{H}_{1} ; \mathscr{H}_{2}\right),\|\cdot\|_{\mathrm{cb}}\right)$ is complete, we are done.

The following definition will allow us to view any s.n. ideal as a space of completely bounded maps. Let $\Phi$ be an s.n. function, and let $\mathscr{H}_{\text {mon }}$ be an arbitrary homogeneous m.c.n. space. We define a new system of matrix norms 
on $\mathscr{H}$ by

$$
\left\|\left(f_{i j}\right)\right\|_{\Phi, \mathrm{mcn}}=\sup _{|T|_{\Phi} \leq 1}\left\|\left(T f_{i j}\right)\right\|_{\mathrm{mcn}} .
$$

Thus given $\left(f_{i j}\right) \in M_{n}(\mathscr{H})$, the new norm is obtained by thinking of $\left(f_{i j}\right)$ as an operator from $\mathfrak{C}_{\Phi}$ to $M_{n}\left(\mathscr{H}_{\mathrm{mcn}}\right)$. Actually, the same number is attained if one merely takes the supremum over all finite rank $T$ with $|T|_{\Phi} \leq 1$. It is easily verified that $\|\cdot\|_{\Phi, \text { mon }}$ is a matrix cross norm, and the resulting m.c.n. space is denoted $\mathscr{H}_{\Phi, \mathrm{mcn}}$. If $\mathscr{H}_{\mathrm{mcn}}$ is an operator space, then so is $\mathscr{H}_{\Phi, \mathrm{mcn}}$. Finally, note that $\mathscr{H}_{\Phi, \text { men }}$ is also homogeneous.

1.3. Proposition. If $\Phi$ is any s.n. function and $\mathscr{H}_{\mathrm{mcn}}$ is any m.c.n. space, then

$$
\mathscr{H}_{\Phi_{1}, \mathrm{mcn}}=\mathscr{H}_{\Phi, \min }=\mathscr{H}_{\text {min }}
$$

and

$$
\mathscr{H}_{\Phi_{\infty}, \mathrm{mcn}}=\mathscr{H}_{\mathrm{mcn}} .
$$

Proof. We will prove that $\mathscr{H}_{\Phi_{1}}$, mcn $=\mathscr{H}_{\text {min }}$ and leave the remaining (elementary) equalities to the reader. Recall that every linear map defined on $\mathfrak{C}_{1}$ attains its norm on rank one elements of $\mathfrak{C}_{1}$. It follows that for $\left(f_{i j}\right) \in M_{n}(\mathscr{H})$ we have

$$
\left\|\left(f_{i j}\right)\right\|_{\Phi_{1}, \text { mcn }}=\sup _{|T|_{1} \leq 1}\left\|\left(T f_{i j}\right)\right\|_{\mathrm{mcn}}=\sup _{\|g\|,\|h\| \leq 1}\left\|\left(\left\langle f_{i j}, h\right\rangle g\right)\right\|_{\mathrm{mcn}}=\left\|\left(f_{i j}\right)\right\|_{\min } .
$$

Proposition 1.3 allows us to think of $\mathscr{H}_{\Phi, \text { men }}$ as a family of interpolating m.c.n. spaces, starting at $\mathscr{H}_{\text {min }}$ and ending at $\mathscr{H}_{\text {mcn }}$. Within this setup there is a linear interpolation of $\mathscr{H}_{\min }$ to $\mathscr{H}_{\text {mcn }}$, obtained by restricting the s.n. functions to the family $\Phi_{p}$, for $1 \leq p \leq \infty$. We will see this method of interpolation is very different from the method recently introduced by G. Pisier in [6].

\section{HoMOgENEOUS HilbERTIAN OPERATOR SPACES}

We will be interested primarily in the spaces $\mathscr{H}_{\Phi, \max }$. The reader will undoubtedly see places in later sections where more generality may be obtained by substituting in an arbitrary homogeneous Hilbertian operator space for the role played by $\mathscr{K}_{\max }$.

2.1. Proposition. The s.n. ideal $\mathrm{CB}\left(\mathscr{H}_{\min } ; \mathscr{H}_{\max }\right)$ equals $\mathfrak{C}_{1}$ as a set and, consequently, the c.b. norm on $\mathrm{CB}\left(\mathscr{H}_{\min } ; \mathscr{H}_{\max }\right)$ is equivalent to the trace norm. However, this c.b. norm is not equal to the trace norm.

Proof. It suffices to prove that the c.b. norm and the trace norm are equivalent on the finite rank positive operators. Assume that

$$
T=\operatorname{diag}\left(\alpha_{0}, \alpha_{1}, \ldots, \alpha_{n-1}, 0,0, \ldots\right)
$$

relative to an orthonormal basis $\left\{e_{i}\right\}_{i=0}^{\infty}$, and let $U$ be the unitary operator defined by

$$
U e_{i}= \begin{cases}e_{i+1} \bmod n & \text { if } i<n, \\ e_{i} & \text { if } i \geq n\end{cases}
$$

If $I_{n}$ is the projection onto the span of $\left\{e_{i}\right\}_{i=0}^{n-1}$, then

$$
\begin{aligned}
|T|_{1}\left|I_{n}\right|_{\mathrm{cb}} & =\operatorname{tr}(T)\left|I_{n}\right|_{\mathrm{cb}} \\
& =\left|T+U^{-1} T U+\cdots+U^{-(n-1)} T U^{(n-1)}\right|_{\mathrm{cb}} \leq n|T|_{\mathrm{cb}} .
\end{aligned}
$$


By [5, Theorem 2.16], we have $\frac{n}{2} \leq\left|I_{n}\right|_{\mathrm{cb}}$. It follows that

$$
|\cdot|_{1} \leq 2|\cdot|_{\mathrm{cb}} \text {. }
$$

Since the trace norm dominates all other symmetric norms, we have established the equivalence of these two norms.

The fact that these norms are not equal also follows [5, Theorem 2.16], since with $I_{n}$ as above, we have

$$
\left|I_{n}\right|_{\mathrm{cb}} \leq n / \sqrt{2}<\left|I_{n}\right|_{1}
$$

Given an s.n. function $\Phi$, let $\Phi_{\mathrm{cb}}$ denote the s.n. function obtained from the s.n. ideal $\mathrm{CB}\left(\mathscr{H}_{\Phi, \max } ; \mathscr{H}_{\max }\right)$.

2.2. Theorem. Let $\Phi$ be an arbitrary s.n. function. Then $\mathrm{CB}\left(\mathscr{H}_{\Phi, \max } ; \mathscr{H}_{\max }\right)$ coincides with $\mathfrak{C}_{\Phi}^{\prime \prime}$ as a set, and $\Phi_{\mathrm{cb}}$ is equivalent to $\Phi$. Furthermore, the s.n. ideal $\mathrm{CB}\left(\mathscr{H}_{\min } ; \mathscr{H}_{\Phi, \max }\right)$ coincides with $\mathfrak{C}_{\Phi^{*}}^{\prime \prime}$ as a set, and the c.b. norm is equivalent to $|\cdot|_{\Phi^{*}}$.

Note that ideals of the form $\mathrm{CB}\left(\mathscr{H}_{1} ; \mathscr{H}_{2}\right)$ are always full (in the sense discussed just before Lemma 1.1). It is a consequence of Lemma 1.1 that the only s.n. functions for which $\mathfrak{C}_{\Phi}$ fails to be full are those that are equivalent to the minimal s.n. function, in which case $\mathfrak{C}_{\Phi}$ is the ideal of compact operators and $\mathfrak{C}_{\Phi}^{\prime \prime}$ is $B(\mathscr{H})$. The double primes that appear in the statement of Theorem 2.2 are needed to take care of the case when either $\Phi$ or $\Phi^{*}$ is equivalent to the minimal s.n. function. In all other cases we have $\mathfrak{C}_{\Phi}=\mathfrak{C}_{\Phi}^{\prime \prime}$ so the double prime may be removed.

Proof. First note that $\Phi_{\mathrm{cb}} \leq \Phi$. This may be seen by assuming $|T|_{\Phi} \leq 1$ and proving $|T|_{\Phi_{\mathrm{cb}}} \leq 1$. Thus assume $|T|_{\Phi} \leq 1$ and $\left\|\left(f_{i j}\right)\right\|_{\Phi, \max } \leq 1$. It follows that

$$
\left\|\left(T f_{i j}\right)\right\|_{\max } \leq 1
$$

so that $T$ is completely contractive; i.e., $|T|_{\Phi_{\mathrm{cb}}} \leq 1$. We deduce from this that

$$
\mathfrak{C}_{\Phi}^{\prime \prime} \subseteq \mathrm{CB}\left(\mathscr{H}_{\Phi, \max } ; \mathscr{H}_{\max }\right) \text {. }
$$

(Recall the relationship between $\mathfrak{C}_{\Phi}^{\prime}$ and $\mathfrak{C}_{\Phi^{*}}$ given in Lemma 1.1.) Now assume that $T \in \mathrm{CB}\left(\mathscr{H}_{\Phi, \max } ; \mathscr{H}_{\max }\right)$. For any $S \in \mathfrak{C}_{\Phi}^{\prime}$ with $|S|_{\Phi^{*}} \leq 1$ and any $R \in \mathfrak{C}_{\Phi}$ with $|R|_{\Phi} \leq 1$ we have $|R S|_{1} \leq 1$. It follows that, as an element of $\mathrm{C} B\left(\mathscr{H}_{\min } ; \mathscr{H}_{\max }\right), R S$ is completely contractive. Since this is true for every such $R$ we see that

$$
\left\|\left(S f_{i j}\right)\right\|_{\Phi, \max }=\sup _{|R|_{\Phi} \leq 1}\left\|\left(R S f_{i j}\right)\right\|_{\max } \leq\left\|\left(f_{i j}\right)\right\|_{\min } .
$$

Thus $S$ is completely contractive as a map in $\mathrm{C} B\left(\mathscr{H}_{\min } ; \mathscr{H}_{\Phi, \max }\right)$. It follows that $T S$ is completely bounded from $\mathscr{H}_{\min }$ to $\mathscr{H}_{\max }$; thus $T S \in \mathfrak{C}_{1}$ by Proposition 2.1. Since $S$ was an arbitrary element of $\mathfrak{C}_{\Phi}^{\prime}$ we have $T \in \mathfrak{C}_{\Phi}^{\prime \prime}$.

We omit the proof of the second assertion, which proceeds similar to the one just given.

For contrast, we have the following two theorems. The proof of the second one follows from the first, in much the same way we deduced Theorem 2.2 from Proposition 2.1. 
2.3. Proposition. The s.n. ideal $\mathrm{CB}\left(\mathscr{H}_{\min } ; \mathscr{H}_{\text {proj }}\right)$ coincides with the ideal of trace class operators, and the trace norm equals the cb norm on $\mathrm{CB}\left(\mathscr{L}_{\min } ; \mathscr{H}_{\text {proj }}\right)$. Proof. Since the trace is the maximal symmetric norm, we have

$$
\mathfrak{C}_{1} \subseteq \mathrm{C} B\left(\mathscr{H}_{\min } ; \mathscr{H}_{\text {proj }}\right) \text {. }
$$

Thus we need only prove that

$$
|T|_{1} \leq|T|_{\mathrm{cb}}
$$

for all finite rank operators $T$. Since both norms are unitary invariant, we may assume that $T$ is positive. Let $\left\{e_{1}, e_{2}, \ldots, e_{n}\right\}$ be an orthonormal set of eigenvectors of $T$ such that $T x=0$ for all $x \in\left\{e_{1}, e_{2}, \ldots, e_{n}\right\}^{\perp}$ and assume $T e_{i}=\alpha_{i} e_{i}($ for $i=1,2, \ldots, n)$. Define a bilinear map

$$
\tau: \mathscr{H} \times M_{n} \rightarrow \mathscr{H}
$$

by

$$
\tau\left(f,\left(a_{i j}\right)\right)=\sum_{i, j=1}^{n} a_{i j}\left\langle f, e_{j}\right\rangle \boldsymbol{e}_{i} .
$$

It follows that $\left\|\tau\left(f,\left(a_{i j}\right)\right)\right\| \leq\|f\|\left\|\left(a_{i j}\right)\right\|$ for all $f \in \mathscr{H}$ and $\left(a_{i j}\right) \in M_{n}$. Thus $\tau$ may be used to define a contraction $\hat{\tau}$ on the projection tensor product via

$$
\hat{\tau}\left(\sum_{i} f_{i} \otimes A_{i}\right)=\sum_{i} \tau\left(f_{i}, A_{i}\right) .
$$

We see that

$$
\begin{aligned}
\sum_{i=1}^{n} \alpha_{i} & =\left\|\hat{\tau}\left(\sum_{n=1}^{n} \alpha_{i} e_{i} \otimes E_{1 i}\right)\right\| \\
& \leq\left\|\sum_{i=1}^{n} \alpha_{i} e_{i} \otimes E_{1 i}\right\| \\
& =\left\|\left(T e_{1} T e_{2} \cdots T e_{n}\right)\right\|_{\text {proj }} \leq|T|_{\mathrm{cb}}
\end{aligned}
$$

(the last inequality follows from the fact that $\left.\left\|\left(e_{1} e_{2} \cdots e_{n}\right)\right\|_{\min }=1\right)$. This completes the proof.

2.4. Theorem. Let $\Phi$ be an arbitrary s.n. function. Then $\mathrm{CB}\left(\mathscr{H}_{\Phi}\right.$, proj $)$ coincides with $\mathfrak{C}_{\Phi}^{\prime \prime}$, and the c.b. norm coincides with $|\cdot|_{\Phi}$. Furthermore, the s.n. ideal $\mathrm{CB}\left(\mathscr{H}_{\min } ; \mathscr{H}_{\Phi, \text { proj }}\right)$ coincides with $\mathfrak{C}_{\Phi}^{\prime \prime}$, and the c.b. norm coincides with $|\cdot|_{\Phi^{*}}$.

The connection between operator ideals and families of completely bounded maps lets us carry results from the former field to the latter. The following two corollaries are samples.

2.5. Corollary. There exist homogeneous Hilbertian operator spaces $\mathscr{H}_{1}$ and $\mathscr{H}_{2}$ with the property that $\mathrm{CB}\left(\mathscr{K}_{1} ; \mathscr{H}_{2}\right) \subset \mathfrak{C}_{\infty}$, but the finite rank operators are not dense (relative to the c.b. norm) in $\mathrm{CB}\left(\mathscr{H}_{1} ; \mathscr{H}_{2}\right)$.

2.6. Corollary. The space $\mathrm{CB}\left(\mathscr{H}_{1} ; \mathscr{H}_{2}\right)$ is separable if and only if the finite rank operators are dense in $\mathrm{CB}\left(\mathscr{H}_{1} ; \mathscr{H}_{2}\right)$.

To prove the first corollary, let $\Phi$ be an s.n. function for which $\mathfrak{C}_{\Phi}$ has the stated property. Then $\mathrm{CB}\left(\mathscr{H}_{\Phi, \max } ; \mathscr{H}_{\max }\right)$ also has the property. Corollary 2.6 follows from [3, Theorem 6.2] (see pp. 89-90 there). 
We would like to point out a consequence of Proposition 1.3; the s.n. function induced by the c.b. norm on $\mathrm{C} B\left(\mathscr{H}_{\min } ; \mathscr{H}_{\max }\right)$ equals $\left(\Phi_{1}\right)_{\mathrm{cb}}$. Thus, in the terminology of [5], the number

$$
\alpha\left(l_{2}(n)\right)
$$

is encoded in the function $\left(\Phi_{1}\right)_{\mathrm{cb}}$. In fact, if $\zeta_{n}$ is the sequence that assigns the constant value 1 for the first $n$ terms and is constantly zero for all remaining terms, then

$$
\alpha\left(l_{2}(n)\right)=\left(\Phi_{1}\right)_{\mathrm{cb}}\left(\zeta_{n}\right) .
$$

\section{A LATtice ViewPoint}

Given an operator space $\mathscr{X}$, we let $\mathscr{X}^{*}$ denote the operator dual of $\mathscr{Z}$ (see [1]). Let $\mathfrak{F}_{\mathscr{K}}$ denote the family of homogeneous Hilbertian operator spaces that have the common base $\mathscr{H}$. It is easy to verify that the operator dual of a space in $\mathfrak{F}_{\mathscr{H}}$ is again in $\mathfrak{F}_{\mathscr{H}}$. If $\mathscr{H}_{\lambda} \in \mathfrak{F}_{\mathscr{H}}(\lambda \in \Lambda)$ are given, then we may define new matrix norms as

$$
\left\|\left(f_{i j}\right)\right\|_{\bigvee_{\lambda} \mathscr{Z}_{\lambda}}=\sup _{\lambda \in \Lambda}\left\|\left(f_{i j}\right)\right\|_{\lambda}
$$

It is easily verified that the resulting space, which we denote $\bigvee \mathscr{H}_{\lambda}$, is in $\mathfrak{F}_{\mathscr{H}}$. Similarly, we may define the meet of the family $\left\{\mathscr{K}_{\lambda}\right\}$ by

$$
\bigwedge \mathscr{H}_{\lambda}=\left(\bigvee \mathscr{L}_{\lambda}^{*}\right)^{*}
$$

It follows that $\mathfrak{F}_{\mathscr{H}}$ is a complete lattice with these lattices operations. If we have $\mathscr{H}_{1}, \mathscr{H}_{2} \in \mathfrak{F}_{\mathscr{H}}$, then we will write $\mathscr{H}_{1} \leq \mathscr{H}_{2}$ if $\left\|\left(f_{i j}\right)\right\|_{1} \leq\left\|\left(f_{i j}\right)\right\|_{2}$ for all $\left(f_{i j}\right) \in M_{n}(\mathscr{H})$ and all $n$. Note that $\bigvee \mathscr{H}_{\lambda}$ is the smallest element of $\mathfrak{F}_{\mathscr{H}}$ bigger than the family $\left\{\mathscr{H}_{\lambda}\right\}$, and $\Lambda \mathscr{H}_{\lambda}$ is the largest element of $\mathfrak{F}_{\mathscr{H}}$ smaller than the family $\left\{\mathscr{H}{ }_{\lambda}\right\}$.

Let $\mathfrak{S}$ be the family of all s.n. functions. It is clear that $\mathfrak{S}$ is also a complete lattice, where the join of a family of s.n. functions is the smallest s.n. function which dominates the family. The meet may be defined using conjugate s.n. functions (just as we did above for homogeneous Hilbert spaces), or we may simply take the join of the set of lower bounds of the family. We let $\mathfrak{S}_{c b}$ denote the set of s.n. functions $\Phi$ for which there exist a homogeneous Hilbertian operator space $\mathscr{H}_{0}$ such that $|\cdot|_{\Phi}$ is equal to the cb norm on $\mathrm{CB}\left(\mathscr{H}_{0} ; \mathscr{H}_{\max }\right)$.

3.1. Lemma. Assume that $\Phi \in \mathfrak{S}_{\mathrm{cb}}$ and $\mathscr{L}_{0} \in \mathfrak{F}_{\mathscr{H}}$ is a space for which $|\cdot|_{\Phi}$ is the c.b. norm on $\mathrm{C} B\left(\mathscr{H}_{0} ; \mathscr{H}_{\text {max }}\right)$. Then we have

$$
\mathscr{H}_{\Phi, \max } \leq \mathscr{H}_{0}
$$

and $\Phi=\Phi_{\mathrm{cb}}$. In particular, for any $\Phi \in \mathfrak{S}$ we have $\left(\Phi_{\mathrm{cb}}\right)_{\mathrm{cb}}=\Phi_{\mathrm{cb}}$.

Proof. Assume that $\left\|\left(f_{i j}\right)\right\|_{0} \leq 1$. Then for any complete contraction $T$ we have $\left\|\left(T f_{i j}\right)\right\|_{\max } \leq 1$, so

$$
\left\|\left(f_{i j}\right)\right\|_{\boldsymbol{\Phi}, \max }=\sup _{\|T\|_{\Phi} \leq 1}\left\|\left(T f_{i j}\right)\right\|_{\max } \leq 1 .
$$

It follows that $\mathscr{H}_{\Phi, \max } \leq \mathscr{H}_{0}$.

As in the proof of Theorem 2.2 we have $\Phi_{\mathrm{cb}} \leq \Phi$. To see the opposite inequality, assume that $|T|_{\Phi_{\mathrm{cb}}} \leq 1$. Since

$$
\mathscr{H}_{\Phi, \max } \leq \mathscr{H}_{0} \leq \mathscr{H}_{\text {max }}
$$


and we are assuming $T$ is completely contractive when viewed as a map from $\mathscr{H}_{\Phi, \max }$, to $\mathscr{H}_{\max }$, it follows that $T$ is completely contractive when viewed from $\mathscr{H}_{0}$ to $\mathscr{H}_{\max }$, i.e., that $|T|_{\Phi} \leq 1$. Thus we have $\Phi \leq \Phi_{\mathrm{cb}}$. The equality $\left(\Phi_{\mathrm{cb}}\right)_{\mathrm{cb}}=\Phi_{\mathrm{cb}}$ follows from the fact that $\Phi_{\mathrm{cb}} \in \mathfrak{S}_{\mathrm{cb}}$ for all $\Phi \in \mathfrak{S}$.

3.2. Proposition. If $\Phi \in \mathfrak{S}$, then

$$
\Phi_{\mathrm{cb}}=\bigvee\left\{\Psi \in \mathfrak{S}_{\mathrm{cb}} \mid \Psi \leq \Phi\right\}
$$

Thus we have that $\mathfrak{S}_{\mathrm{cb}}$ is closed under arbitrary join.

Proof. If $\Psi \in \mathfrak{S}_{\mathrm{cb}}$ and $\Psi \leq \Phi$, then $\Psi=\Psi_{\mathrm{cb}} \leq \Phi_{\mathrm{cb}}$ and

$$
\bigvee\left\{\Psi \in \mathfrak{S}_{\mathrm{cb}} \mid \Psi \leq \Phi\right\} \leq \Phi_{\mathrm{cb}}
$$

The opposite inequality follows from the fact that $\Phi_{\mathrm{cb}} \in\left\{\Psi \in \mathfrak{S}_{\mathrm{cb}} \mid \Psi \leq \Phi\right\}$.

3.3. Theorem. If $\Phi_{\lambda} \in \mathfrak{S}$, then

$$
\bigvee_{\lambda} \mathscr{H}_{\Phi_{\lambda}, \max }=\mathscr{H}_{\bigwedge \Phi_{\lambda}, \max } .
$$

Proof. We will use the fact that $\Psi \leq \Phi$ implies $\mathscr{H}_{\Phi, \max } \leq \mathscr{H}_{\Psi, \max }$. Let $\Phi$ be the s.n. function induced by the c.b. norm on $\mathrm{C} B\left(\bigvee \mathscr{H}_{\Phi_{\lambda}, \max } ; \mathscr{H}_{\max }\right)$. Since

$$
\mathscr{H}_{\Phi_{\lambda}, \max } \leq \bigvee \mathscr{H}_{\Phi_{\lambda}, \max },
$$

we have

$$
\Phi \leq\left(\Phi_{\lambda}\right)_{\mathrm{cb}} \leq \Phi_{\lambda}
$$

for all $\lambda$. It follows that $\Phi \leq \bigwedge_{\lambda} \Phi_{\lambda} \leq \Phi_{\lambda}$ and

$$
\mathscr{H}_{\Phi_{\lambda}, \max } \leq \mathscr{H}_{\Lambda_{\lambda} \Phi_{\lambda}, \max } \leq \mathscr{H}_{\Phi, \max } \leq \bigvee_{\lambda} \mathscr{H}_{\Phi_{\lambda}, \max }
$$

(The rightmost inequality follows from Lemma 3.1.) Since the join is the smallest space bigger than all the $\mathscr{H}_{\Phi_{\lambda}, \max }$, we conclude that

$$
\mathscr{H}_{\Lambda_{\lambda} \Phi_{\lambda}, \max }=\mathscr{H}_{\Phi, \max }=\bigvee_{\lambda} \mathscr{H}_{\Phi_{\lambda}, \max } .
$$

We wish to illustrate Theorem 3.3 with the following example. For $0<r<1$ let $\Psi_{r}$ be the s.n. function defined on $\hat{\mathbf{k}}$ by

$$
\Psi_{r}(\zeta)=\sum_{n} r^{n} \zeta_{n}
$$

(See [3, p. 145].) It is not hard to see that $\Psi_{r}$ is equivalent to $\Phi_{\infty}$ for all $0<r<1$. It is also apparent that $\bigvee \Psi_{r}=\Phi_{1}$. It follows that the spaces $\mathscr{H}_{\Psi_{r}, \text { max }}$ are all completely isomorphic to $\mathscr{H}_{\min }$ and

$$
\bigvee_{r} \mathscr{H}_{\Psi_{r}, \max }=\mathscr{H}_{\text {max }} \text {. }
$$

It is not hard to modify this procedure to obtain an arbitrary homogeneous Hilbertian operator space as the join of spaces completely isomorphic to $\mathscr{H}_{\text {min }}$. It follows that, given any positive number $r$, it is possible to find a homogeneous Hilbertian operator space $\mathscr{H}_{0}$ whose c.b. distance to $\mathscr{H}_{\min }$ is a finite number greater than $r$ (see [5] for a definition of c.b. distance). 
The results above give a picture of where the spaces $\mathscr{H}_{\Phi, \max }$ live, relative to other Hilbertian operator spaces. If $\Phi \in \mathfrak{S}_{\mathrm{cb}}$, then there are many candidates $\mathscr{H}_{0} \in \mathfrak{F}_{\mathscr{H}}$ for which $\Phi$ will be the s.n. function corresponding to the c.b. norm on $\mathrm{CB}\left(\mathscr{H}_{0} ; \mathscr{H}_{\max }\right)$. It follows from Lemma 3.1 that $\mathscr{H}_{\Phi, \max }$ is the smallest such space. Since the operator dual reverses an inequality relating two spaces, we may also note that $\mathscr{H}_{\Phi, \max }^{*}$ is the largest space among the spaces $\mathscr{H}_{0}$ that have $\Phi$ as the s.n. function induced by the c.b. norm on $\mathrm{CB}\left(\mathscr{H}_{\min } ; \mathscr{H}_{0}\right)$.

\section{The CENTRAL SPACES}

We will say that a homogeneous Hilbertian operator space $\mathscr{H}_{0}$ is a central space if the sets $\mathrm{CB}\left(\mathscr{H}_{\min } ; \mathscr{H}_{0}\right)$ and $\mathrm{CB}\left(\mathscr{H}_{0} ; \mathscr{H}_{\max }\right)$ both coincide with the set of Hilbert-Schmidt operators. We let $\mathscr{H}_{\text {col }}$ and $\mathscr{H}_{\text {row }}$ denote column and row Hilbert space (respectively). It is known that $\mathscr{H}_{\text {col }}$ and $\mathscr{H}_{\text {row }}$ are not only central spaces, but as s.n. ideals

$$
\begin{aligned}
\mathfrak{C}_{2} & =\mathrm{CB}\left(\mathscr{H}_{\min } ; \mathscr{H}_{\text {col }}\right)=\mathrm{CB}\left(\mathscr{H}_{\text {col }} ; \mathscr{H}_{\text {max }}\right) \\
& =\mathrm{C} B\left(\mathscr{H}_{\text {min }} ; \mathscr{H}_{\text {row }}\right)=\mathrm{C} B\left(\mathscr{H}_{\text {row }} ; \mathscr{H}_{\text {max }}\right)
\end{aligned}
$$

isometrically. (See [4] for an account of this.)

Recall that we denote the Hilbert-Schmidt norm by $|\cdot|_{2}$, and the corresponding s.n. function $\Phi_{2}$ is the 2-norm. It follows from Lemma 3.1 that $\mathrm{CB}\left(\mathscr{H}_{\Phi_{2}}\right.$, max $\left.; \mathscr{H}_{\max }\right)$ is also the s.n. ideal of Hilbert-Schmidt operators. Thus $\mathscr{H}_{\Phi_{2}, \max }$ is a central space by Theorem 2.2. Note that Theorem 2.2 only tells us that $\mathrm{CB}\left(\mathscr{H}_{\min } ; \mathscr{H}_{\Phi_{2}}\right.$,max $)$ is equal to $\mathfrak{C}_{2}$ as a set, with an equivalent (but in general not equal) norm. In fact, we will soon see that the c.b. norm on $\mathrm{CB}\left(\mathscr{H}_{\min } ; \mathscr{H}_{\Phi_{2}, \text { min }}\right)$ cannot equal the Hilbert-Schmidt norm.

\subsection{Proposition. Assume the spaces $\mathscr{H}_{1}$ and $\mathscr{H}_{2}$ are central spaces and}

$$
\mathscr{H}_{1} \leq \mathscr{H}_{0} \leq \mathscr{H}_{2}
$$

Then $\mathscr{H}_{0}$ is also central.

Proof. If $T$ is Hilbert-Schmidt, then $T \in \mathrm{CB}\left(\mathscr{H}_{\min } ; \mathscr{H}_{2}\right)$. The assumed inequality then implies $T \in \mathrm{CB}\left(\mathscr{H}_{\min } ; \mathscr{H}_{0}\right)$. Thus $\mathfrak{C}_{2} \subset \mathrm{CB}\left(\mathscr{H}_{\min } ; \mathscr{H}_{0}\right)$. Conversely, if $T \in \mathrm{CB}\left(\mathscr{H}_{\min } ; \mathscr{H}_{0}\right)$, then the inequality gives us $T \in \mathrm{CB}\left(\mathscr{H}_{\min } ; \mathscr{H}_{1}\right)$ whence $T$ is Hilbert-Schmidt. Thus $\mathfrak{C}_{2}=\mathrm{CB}\left(\mathscr{H}_{\min } ; \mathscr{H}_{0}\right)$ (as a set), and the equality of sets $\mathfrak{C}_{2}=\mathrm{C} B\left(\mathscr{H}_{0} ; \mathscr{H}_{\max }\right)$ is proven similarly.

Since the operator dual of a central space is again central, Proposition 4.1 tells us that the interval of spaces

$$
\left[\mathscr{H}_{\Phi_{2}, \max } ; \mathscr{H}_{\Phi_{2}, \text { max }}^{*}\right]=\left\{H_{0} \mid \mathscr{H}_{\Phi_{2}, \max } \leq \mathscr{H}_{0} \leq \mathscr{H}_{\Phi_{2}, \max }^{*}\right\}
$$

consists of central spaces. Lemma 3.1 and the duality relationship between $\mathscr{H}_{\text {col }}$ and $\mathscr{H}_{\text {row }}$ show that $\mathscr{H}_{\text {col }}, \mathscr{H}_{\text {row }} \in\left[\mathscr{H}_{\Phi_{2}, \max } ; \mathscr{H}_{\Phi_{2}}^{*}\right.$, max $]$, whence we conclude that

$$
\mathscr{H}_{\text {col }} \vee \mathscr{H}_{\text {row }}, \mathscr{H}_{\text {col }} \wedge \mathscr{H}_{\text {row }} \in\left[\mathscr{H}_{\Phi_{2}, \text { max }} ; \mathscr{H}_{\Phi_{2}, \text { max }}^{*}\right] \text {. }
$$

In [6] Pisier construct a homogeneous Hilbertian operator space $\mathscr{H}_{\text {oh }}$ that is self-dual, and it is seen in this paper (Corollary 2.6 of our version) that

$$
\mathscr{H}_{\text {col }} \wedge \mathscr{H}_{\text {row }} \leq \mathscr{H}_{\text {oh }} \leq \mathscr{H}_{\text {col }} \vee \mathscr{H}_{\text {row }}
$$

so $\mathscr{H}_{\text {oh }}$ is central. 
At first glance, one might guess (as we did) that

$$
\mathscr{H}_{\Phi_{2}, \max }=\mathscr{H}_{\text {col }} \wedge \mathscr{H}_{\text {row }} \text {. }
$$

We prove that these matrix norms are not completely boundedly equivalent by computing the norm of an $n \times n$ matrix $\left(e_{i j}\right)$, with the doubly indexed set $\left\{e_{i j}\right\}$ forming an orthonormal basis. A direct computation reveals that

$$
\left\|\left(e_{i j}\right)\right\|_{\mathscr{K}_{\text {col }} \vee \mathscr{H}_{\text {row }}}=\sqrt{n} .
$$

Using the fact that $\mathscr{H}_{\text {col }} \vee \mathscr{H}_{\text {row }}$ is the operator dual of $\mathscr{H}_{\text {col }} \wedge \mathscr{H}_{\text {row }}$ we have

$$
n=\left\|\left(\left\langle e_{k l}, e_{i j}\right\rangle_{i, j, k, l=1}^{n}\right)\right\| \leq\left\|\left(e_{i j}\right)\right\|_{\mathscr{H}_{\text {col }} \wedge \mathscr{H}_{\text {row }}} \sqrt{n} .
$$

It follows that $\sqrt{n} \leq\left\|\left(e_{i j}\right)\right\|_{\mathscr{H}_{\text {col }} \wedge \mathscr{\not}_{\text {row }}}$. But it is easy to see that $\left\|\left(e_{i j}\right)\right\|_{\Phi_{2}, \max } \leq 1$, since if $T$ is a Hilbert-Schmidt contraction one has

$$
\sum_{i, j=1}^{n}\left\|T e_{i j}\right\|^{2} \leq 1 .
$$

We mentioned earlier that although $\mathscr{H}_{\Phi_{2}, \max }$ is a central space, it cannot be that the c.b. norm on $\mathrm{CB}\left(\mathscr{H}_{\min } ; \mathscr{H}_{\Phi_{2}, \max }\right)$ is equal to the Hilbert-Schmidt norm. We wish now to justify this assertion. If it were equal, then $\mathscr{H}_{\Phi_{2}}^{*}$, max would have the property that the c.b. norm in both $\mathrm{C} B\left(\mathscr{H}_{\min } ; \mathscr{H}_{\Phi_{2} \text {, max }}^{*}\right)$ and

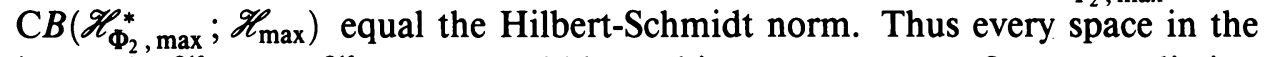
interval $\left[\mathscr{H}_{\Phi_{2}, \max } ; \mathscr{H}_{\Phi_{2}}^{*}\right.$, max $]$, would have this same property. Our contradiction arises by observing that Pisiers space $\mathscr{H}_{\mathrm{oh}}$ cannot have this property. Indeed, assume that $\mathscr{H}$ is $n$ dimensional and $\left\{e_{i}\right\}$ is an orthonormal basis of $\mathscr{H}$, and let $\beta$ be the c.b. norm of the identity map in $\mathrm{C} B\left(H_{\min } ; \mathscr{H}_{\mathrm{oh}}\right)$. Then

$$
\begin{aligned}
\beta & =\sup \left\{\left\|\sum e_{i} \otimes A_{i}\right\|_{\mathrm{oh}} \mid\left\|\sum e_{i} \otimes A_{i}\right\|_{\min } \leq 1\right\} \\
& =\sup \left\{\left\|\sum A_{i} \otimes \bar{A}_{i}\right\|^{1 / 2} \mid\left\|\sum e_{i} \otimes A_{i}\right\|_{\min } \leq 1\right\} \\
& \leq \sup \left\{\left\|\sum A_{i} \otimes B_{i}\right\|^{1 / 2} \mid\left\|\sum e_{i} \otimes A_{i}\right\|_{\min },\left\|\sum e_{i} \otimes B_{i}\right\|_{\min } \leq 1\right\} \\
& =\alpha\left(l_{2}(n)\right)^{1 / 2}<\sqrt{n} .
\end{aligned}
$$

(The second equality may be found in the first section of [6], and the last equality follows from [5, Proposition 2.3] and its preceding remarks.) Thus the Hilbert-Schmidt norm does not agree with the c.b. norm on the identity. A little more thought will convince the reader that the s.n. functions induced by the c.b. norms on $\mathrm{CB}\left(\mathscr{H}_{\min } ; \mathscr{H}_{\text {oh }}\right)$ and $\mathrm{CB}\left(\mathscr{H}_{\text {oh }} ; \mathscr{H}_{\max }\right)$ are the same (because of duality relations) and

$$
\beta=\alpha\left(l_{2}(n)\right)^{1 / 2} .
$$

\section{REFERENCES}

1. D. P. Blecher and V. I. Paulsen, Tensor products of operator spaces, J. Funct. Anal. 99 (1991), 262-292.

2. E. G. Effros and Z.-J. Ruan, On matrically normed spaces, Pacific J. Math. 132 (1988), 243-264. 
3. I. C. Gohberg and M. G. Krein, Introduction to the theory of linear nonselfadjoint operators, Transl. Math. Monographs, vol. 18, Amer. Math. Soc., Providence, RI, 1969.

4. B. Mathes, Characterizations of row and column Hilbert space, J. London Math. Soc. (2) 50 (1994), 199-208.

5. V. I. Paulsen, Representations of function algebras, abstract operator spaces, and Banach space geometry, J. Funct. Anal. 109 (1992), 113-129.

6. G. Pisier, The operator Hilbert space $\mathrm{OH}$, complex interpolation and tensor norms, preprint.

7. Z.-J. Ruan, Subspaces of $C^{*}$-algebras, J. Funct. Anal. 76 (1988), 217-230.

Department of Mathematics, Colby College, Waterville, Maine 04901

E-mail address: dbmathes@colby .edu

Department of Mathematics, University of Houston, Houston, Texas 77204-3476

E-mail address: vernomath.uh.edu 